Proyecciones

Vol. $12 \mathrm{~N}^{\circ} 1$, pp.21-33 Junio 1993

Universidad Católica del Norte

Antofagasta - Chile

\title{
AUTO-ACCESIBILIDAD EN SISTEMAS DE CONTROL SINGULARES*
}

\author{
HERNAN HENRIQUEZ M. \\ Universidad de Santiago, Santiago, Chile. \\ GENARO CASTILLO G. \\ Universidad de Talca, Talca, Chile.
}

\section{Resumen}

En este trabajo se presenta un concepto de auto-accesibilidad para sastemas de control lineales singulares y se caracterizan los sistemas auto-accesibles en término de sus parámetros. Además se relaciona la auto-accesibilidad con la estabilidad del sistema y se establece una propiedad geométrica de las trayectorias de auto-accesibilidad.

* Trabajo parcialmente financiado por Proyecto FONDECYT 92-185 y DICYT. Proyecto 04-9133HM. 


\section{Introducción.}

En este trabajo consideraremos sistemas de control lineales descritos pur la ecuación

$$
E \dot{x}(t)=A x(t)+B u(t)
$$

donde $x \in \mathbb{R}^{n}, u \in \mathbb{R}^{m}$ representan el estado $y$ control, respectivarrient. $E, A \in \mathbb{R}^{n \times n}$ y $B \in \mathbb{R}^{n \times m}$ sun matrices constantes El sisterna se denomma normal cuando la matriz $E$ es no singular y singular en caso contrario. La solubilidad de la ecuación ( 1 ) depende del par $(E, A)$ Este se llama regular ruando, $\operatorname{det}(\lambda E-A)$ no es identicamente igual a cero. En este caso el sistema (1) puede. descomponerse, nediante una transformación de coordenadas, en dos subsistemas.

$$
\dot{x}_{1}(t)=A_{1} x_{1}(t)+B_{1} u(t)
$$

$$
V_{x_{2}}(l)=x_{2}(t)+B_{2} u(t)
$$

denoninados sub-sistema lento y sub-sistema rápido. respectivanente. En estas ecuaciones $A_{1} \in \mathbb{R}^{n_{1} \times n_{1}} \quad N \in \mathbb{R}^{n_{2} \times n_{2}} \quad n_{1}+n_{2}=n$ y $\quad N$ es una matriz nilpotente de índice $h$. Como el sub-sistema lento es un sistema normal, su solución se expresia mediante la fórmula de variación de constantes

$$
x_{1}(t)=e^{A_{1} t} x_{1}(0)+\int_{0}^{t} e^{A_{1}(t-s)} B_{1} u(s) d s
$$

mientras que la solución del sub-sistema rápido se obtiene por la fórmula

$$
x_{2}(t)=-\sum_{i=0}^{h-1} N^{i} B_{2} u^{(i)}(t)
$$

donde $u^{(i)}(t)$ denota la $i$-ésima derivada del control $u$. Por este motivo restringiremos el espacio de controles al conjunto $C_{p}^{h-1}$ formado por las funciones $u(\cdot)$ que son seccionalmente de clase $h-1$. En el desarrollo que sigue usaremos la notación

$$
<A, B>=\operatorname{Im}\left[B, A B, \ldots, A^{n-1} B\right] .
$$

A continuación consideraremos un sistema de control singular regular representado en su forma estandard por las ecuaciones (2)-(3). Por este motivo el vector de estado $x(t)$ se identifica con el par $\left(x_{1}(t) . x_{2}(t)\right)$. 
Definición 1. Un vector $w \in \mathbb{R}^{n}$ se dice alcanzable si existe una condición inicial $x_{1}(0)$, un control admisible $u(\cdot) \in\left(_{p}^{h-1}\right.$ y $t_{1}>0$ tal que $x\left(t_{1}\right)=\left(x_{1}\left(t_{1}\right), x_{2}\left(t_{1}\right)\right)=$ wi

El conjunto

$$
R\left(x_{1}(0)\right)=\left\{w \in \mathbb{R}^{n} \text { existen } \iota_{1}>0 \text { y } u(.) \in C_{p}^{h-1} \text { tal que } x\left(t_{1}\right)=w\right\}
$$

se llama conjunto de alcanzabilidad desde la condicion inicial $x_{1}(0)$. Ha sidr, demostrado en [3] yue

$$
R(0)=\left\langle A_{1} \cdot B_{1}>\uparrow<N, B_{2}\right\rangle
$$

Llamaremos conjunto de accesibilidad a

$$
R=\bigcup_{s_{1}(0) \in \mathbb{R}^{n_{1}}} R\left(x_{1}(0)\right)=\mathbb{R}^{n_{1}} \oplus\left\langle N, B_{2}\right\rangle
$$

La controlabilidad de un sistema singular se define de la misma forma que para los sistemas lineales normales.

Definición 2. El sistema (1) se dice controlable si para todo $\iota_{1}>0$, y todo par de vectores $x(0) \in \mathbb{R}^{n}$ y $w \in \mathbb{R}^{n}$ existe $u(.) \in C_{p}^{h-1}$ tal quo $x\left(t_{1}\right)=w$

El siguiente resultado, cuya demostración puede encontrarse en [3], carac. teriza la controlabilidad de sistemas singulares

Teorema 1. Todo sistema singular regular verifica las siguientes propiedades

(i) El sub-sistema lento es controlable si, y solamente si,

$$
\operatorname{rang}[\lambda E-A, B]=n, \text { para todo } \lambda \in \mathbf{C}
$$

(ii) Las siguientes condiciones son equivalentes

(a) El sub-sistema rápido es controlable.

(b) $\operatorname{rang}\left[B_{2}, N B_{2}, \quad . N^{h-1} B_{2}\right]=n_{2}$.

(c) $\operatorname{rang}\left[N, B_{2}\right]=n_{2}$

(d) $\operatorname{rang}[E, B]=n$ 
(iii) Las siguientes condiciones son equivalentes:

(a) El sistema (1) es controlable.

(b) Los sub-sistemas lento y rápido son controlables.

(c) $\operatorname{rang}\left[B_{1}, A_{1} B_{1}, \ldots, A_{1}^{n_{1}-1} B_{1}\right]=n_{1} \quad$ y $\operatorname{rang}\left[B_{2}, N B_{2}, \ldots, N^{h-1} B_{2}\right]=n_{2}$.

(d) $\operatorname{rang}[\lambda E-A, B]=n$, para todo $\lambda \in \mathbf{C}, \mathrm{y} \operatorname{rang}[E, B]=n$.

Definición 3. El sistena singular (1) se dice $R$-controlable si los estados en $R$ son controlables

Se deduce de los resultados precedentes la siguiente caracterización de la $R$-controlabilidad.

Teorema 2. Las siguientes condiciones son equivalentes

(a) El sistena (1) es $R$-controlable.

(b) El sub-sistema lento es controlable.

(c) $\operatorname{rang}[\lambda E-A, B]=n$, para todo $\lambda \in \mathrm{C}$.

(d) $\operatorname{rang}\left[B_{1}, A_{1} B_{1}, \ldots, A_{1}^{n_{1}-1} B_{1}\right]=n_{1}$.

\section{Auto-accesibilidad de sistemas singulares.}

En esta sección seguimos considerando un sistema de control singular regular descrito por la ecuación (1) y representado en su forma estandard por las ecuaciones (2)-(3).

Cuando un sistema ha sido controlado, esto es, ha sido posicionado ell un estado deseado, nos interesa mantenerlo en ese estado. Normalnente esto, es imposible debido a las perturbaciones que actuan sobre él. Si el sistema es sacado de su estado, la controlabilidad nos garantiza la posibilidad de volverlo al estado inicial, sin embargo esto puede obtenerse también si el sisterra tiene una propiedad más débil que la controlabilidad, la que se denomina auto-accesibilidad. Este concepto fue introducido por A. Baccioti en [I] y posteriormente estudiado por Barbanti [2] para sistemas descritos por ecuaciones integrales y por Henríquez [4] para sistemas lineales distribuidos. A continuación introduciremos un concept") de auto-accesibilidad para sisternas singulares, con el cual podemos extender a este contexto los resultados obtenidos anteriormente en [4] y [5]. 
Defición 4. Un vector $w \in \mathbb{R}^{n}$ se dice auto-accesible en $\left[0, t_{1}\right]$ si existe $u(\cdot) \in C_{p}^{h-1}$ tal que $x(0)=x\left(t_{1}\right)=w$. Diremos también que $w$ es auto-accesible si lo es en el intervalo $\left[0, t_{1}\right]$, para todo $t_{1}>0$.

Defición 5. El sistema de control (1) se llama auto-accesible (respectivarnente. en $\left.\left[0, t_{1}\right]\right)$ si todo estado $w \in \mathbb{R}^{n}$ es auto -accesible (respectivarnente, en $\left[0, t_{1}\right]$ )

De acuerdo a estas definiciones, la propiedad de auto-accesibilidad representa la capacidad del sistema de retornar a un estado inicial. El siguiente teorema caracteriza los estados auto-accesibles.

Lema 1. Para cualquier conjunto de vectores $x_{i}, y_{i} \in \mathbb{R}^{n}, i=0,1, \ldots, h-1, y$ todo par de números $t_{1}, t_{2}$ distintos, existe un polinomio vectorial $f$, con valores en $\mathbb{R}^{n}$ y grado menor o igual a $2 h-1$, tal que $f^{(i)}\left(t_{1}\right)=x_{i}$ y $f^{(i)}\left(t_{2}\right)=y_{i}$ $i=0,1, \ldots, h-1$

Demostración. Sea $t_{0} \in \mathbb{R}$ y $\operatorname{sean} z_{i}, i=0,1, \ldots, h-1$, vectores en $\mathbb{R}^{n}$ Entonces existe un polinomiog de grado $h-1$ tal que $g^{(i)}\left(t_{0}\right)=z_{i}$ Si definimos el polinomio f en la forma

$$
f(t)=\left(t-t_{1}\right)^{h} g_{2}(t)+\left(t-t_{2}\right)^{h} g_{1}(t) \text {. }
$$

usando la idea anterior podemos determinar $g_{1}$ y $g_{2}$ de modo que $f$ verifique las condiciones del enunciado.

Teorema 3. Un vector $w=\left(w_{1}, w_{2}\right) \in \mathbb{R}^{n}$ es auto-accesible si, y solamente sI. $A_{1} w_{1} \in<A_{1}, B_{1}>$ y $w_{2} \in<N, B_{2}>$

Demostración. Si $w$ es auto-accesible entonces para cada tiempo $t>0$ existe un control $u(\cdot)$ para el cual la solución $x(t)$ de la ecuación (2) verifica la relación

$$
w_{1}=x_{1}(t)=e^{A_{1} t} w_{1}+\int_{0}^{t} e^{A_{1}(t-s)} B_{1} u(s) d s
$$

luego

$$
w_{1}-e^{A_{1} t} w_{1}=\int_{0}^{t} e^{A_{1}(t-s)} B_{1} u(s) d s \in\left\langle A_{1}, B_{1}\right\rangle
$$

y en consecuencia

$$
-A_{1} \int_{0}^{t} e^{A_{1} s} w_{1} d s=\int_{0}^{t} e^{A_{1}(t-s)} B_{1} u(s) d s \in\left\langle A_{1}, B_{1}\right\rangle
$$

Definiendo la matriz $M:=-\int_{0}^{t} e^{A_{1} s} d s$ entonces es bien conocido que $M$ es invertible para $0<t<\epsilon$, para cierto $\epsilon>0$ y que el espacio $\left\langle A_{1}, B_{1}>\right.$ es invariante para $M$. Como $A_{1} M w_{1} \in<A_{1}, B_{1}>$ entonces $A_{1} w_{1} \in M^{-1}<A_{1}, B_{1}>\subseteq$ $<A_{1}, B_{1}>$. 
Por olra parte. conto

$$
\left.w_{2}=x_{2}(t)=-\sum_{i=0}^{h-1} N^{i} B_{2} u^{(i)}(l) \in<N B_{2}\right\rangle
$$

obtenemos la afirmación.

Reciprocamente si $A_{1} w_{1} \in<A_{1}, B_{1}>$ y $w_{2} \in<N . B_{2}>$ rutouces. para cada $t_{1}>0$ podemos afirmar que

$$
w_{1}-p_{1}^{A_{1}} w_{1}=A_{1} M w_{1} \in M<A_{1}, B_{1}>\subseteq<A_{1}, B_{1}>
$$

Modificando leventente la demostración del teorema $2-1.1$ de [3], fror fal uso del lema precedente en lugar del lema 2-1.2 utilizado en [3]. podenos alirnar yu el vector $\left(w_{1}-c^{A_{2} t_{1}} w_{1}, w_{2}\right)$ es alcanzable desde $\left(0, w_{2}\right)$ y en consecuencia . existr un control $u(\cdot) \in\left(_{p}^{h-1}\right.$ tal que lat respectiva solución $x(\cdot)$ satisface $r(\theta)=0 y$

$$
\mu\left(t_{1}\right)=\left[\begin{array}{c}
x_{1}\left(t_{1}\right) \\
x_{2}\left(t_{1}\right)
\end{array}\right]=\left[\begin{array}{c}
w_{1}-p^{\lambda_{1} t_{1}} w_{1} \\
w_{2}
\end{array}\right]=\left[\begin{array}{c}
\int_{0}^{t_{1}} e^{A_{1}\left(t_{1}-s\right)} B_{1} u(s) d s \\
-\sum_{i=1}^{h-1} N^{i} B_{2} u^{(i)}\left(t_{1}\right)
\end{array}\right]
$$

Resulta de lo anterior que

$$
w_{1}=r^{A} t_{1} u_{1}+\int_{0}^{t_{1}} e^{A_{1}\left(t_{1}-s\right)} B_{1} u(s) d s
$$

y

$$
w_{2}=-\sum_{i=1}^{n-1} N^{i} B_{2} u^{(i)}\left(t_{1}\right)
$$

lo cual muestra que el vector $w=\left(w_{1}, w_{2}\right)$ es auto-accesible en et intervalo $\left[0, t_{1}\right]$

Podemos ahora establecer el siguiente jesultado.

Corolario 1. Considere las siguientes atimaciones.

(a) El sistema (1) es auto-accesible.

(b) El espacio $\operatorname{Im}\left(A_{1}\right) \subseteq<A_{1}, B_{1}>$ y $\mathbb{R}^{n_{2}}=\left\langle N, B_{2}\right\rangle$

(c) El sub-sisterna lento es auto-accesible y ef sub-sistema rápido es controlable

(d) $\operatorname{rang}[\lambda E-A, B]=n$, pară todo $\lambda \in \mathrm{C}, \lambda \neq 0$ y $\operatorname{rang}[E, B]=n$. 


$$
\text { Entances }(a) \rightarrow(b) \mapsto(c) \rightarrow(d) \text {. }
$$

Demostración. La equivalencia de las condiciones (a), (b) y (c) is consecuencia inmediata del teorema precedente. Para demostrar que cualquiera de estas condiciones implica (d) introduciremos algunas notaciones. Sea $\gamma_{1}$ el subespacio de $\mathbb{R}^{n_{1}}$ formado por los vectores $x_{1}$ tal que $A^{i} x_{1}=0$, para algín $i \geq 1$. Ls bien conocido que existe un número natural $k$ tal que, si $Y_{2}:=\operatorname{Im}\left(A_{1}^{k}\right)$, entonces $\mathbb{R}^{n_{1}}=Y_{1} \oplus Y_{2}$. Si denotamos por $y_{i}:=\pi_{i}\left(x_{1}\right), i=1,2$, las componentes le $x_{1}$ con $Y_{i}$, respectivamente, entonces el subsistema lento se descornpone a su vez ell dos subsistemas

$$
\dot{y}_{1}(t)=A_{11} y_{1}(t)+B_{11} u(t)
$$

$$
y_{2}(t)=A_{12} y_{2}(t)+B_{12} u(t)
$$

doncle $A_{11}$ indica la restricción de $A_{1}$ a $Y_{2}$ y $B_{1 i}:=\pi_{i} B_{1}, i=1,2$. lisaremos también la notación $n_{1 i}$ para la dimension de $Y_{i}$, cle modo que $n_{1}=n_{11}+n_{12}$. En consecuencia, escogiendo bases adecuadas, podemos afirmar qur

$$
\begin{aligned}
\operatorname{rang}[\lambda E-A, B] & =\operatorname{rang}\left[\begin{array}{ccc}
\lambda I_{n_{1}}-A_{1} & 0 & B_{1} \\
0 & \lambda N-I_{n_{2}} & B_{2}
\end{array}\right] \\
& =\operatorname{rang}\left[\begin{array}{cccc}
\lambda I_{n_{11}}-A_{11} & 0 & 0 & B_{11} \\
0 & \lambda I_{n_{12}}-A_{12} & 0 & B_{12} \\
0 & 0 & \lambda N-I_{n_{2}} & B_{2}
\end{array}\right]
\end{aligned}
$$

Si suponemos que el sistema (1) es auto-accesible, entonces $\operatorname{Im}\left(A_{1}\right) \subseteq<$ $A_{1}, B_{1}>$. Resulta de esto que $\operatorname{Im}\left(A_{12}\right) \subseteq\left\langle A_{12}, B_{12}>\right.$ y como $A_{12}$ es un isomorfismo entonces $\mathrm{el}$ sistema (5) es controlable y por el criterio de Hautus $([6])$ podemos afirmar que rang $\left[\lambda I_{n_{12}}-A_{12}, B_{12}\right]=n_{12}$, para todo $\lambda \in \mathrm{C}$. Por otra parte como el único valor propio posible de $A_{11}$ es 0 , entonces rang $\left[\lambda I_{n_{11}}-A_{11}\right]=$ $n_{11}$, todo $\lambda \in \mathrm{C}, \lambda \neq 0$. En consecuencia $\operatorname{rang}[\lambda E-A, B]=n$, para todo $\lambda \neq 0$. Se completa la demostración observando que la controlabilidad del sistema rápido implica que rang $[E, B]=n$.

Es fácil construir ejemplos para verificar que las condición (d) no implica (a). No obstante, si se verifica (d) y además $\left.A_{1}\left(Y_{1}\right) \subseteq<A_{1}, B_{1}\right\rangle$, entonces el sistema (1) es auto- accesible. La situación anterior se presenta, por ejemplo, cuando $Y_{1}=\operatorname{ker} A$, lo que es equivalente a que la multiplicidad algebraica del valor propio 0 de A sea menor o igual a 1 .

El siguiente corolario justifica las afirmaciones efectuadas al inicio de esta sección sobre la propiedad de auto-accesibilidad 
Corolario 2. Sean $0<t_{1}<t_{2}$. Si $w$ es un estado auto- accesible del sisterna (1) y $z=x\left(t_{1}\right)$, siendo $x(\cdot)$ la trayectoria correspondiente a un control admisible $u(\cdot)$ con condición inicial $x(0)=w$, entonces existe un control admisible $v(\cdot)$ definido en $\left[t_{1}, t_{2}\right]$ tal que la trayectoria correspondiente satisface las condiciones $y\left(t_{1}\right)=z$ y $y\left(t_{2}\right)=w$.

Demostración. Supongamos que $w=\left(w_{1}, w_{2}\right)$ y que $z=\left(z_{1}, z_{2}\right)$. Entonces

$$
z_{1}=e^{A_{1} t_{1}} w_{1}+\int_{0}^{t_{1}} e^{A_{1}\left(t_{1}-s\right)} B_{1} u(s) d s .
$$

Como $A_{1} w_{1} \in<A_{1}, B_{1}>$ de las propiedades de invarianza de este espacio sc deduce que $w_{1}-f^{A_{1}\left(t_{2}-t_{1}\right)} z_{1} \in<A_{1}, B_{1}>$. Además tanto $w_{2}$ como $z_{2}$ pertenecell a $\left\langle N, B_{2}\right\rangle$. De las propiedades de controlabilidad de estos espacios se deduce la existencia de un control $v_{1}(\cdot)$ definido en el intervalo $\left[0, t_{2}-t_{1}\right]$ tal que la trayectoria $\tilde{y}(\cdot)$ correspondiente al control $v_{1}$, satisface las condiciones $\tilde{y}(0)=\left(0, z_{2}\right)$ y $\tilde{y}\left(t_{2}-t_{1}\right)=\left(w_{1}-e^{A_{1}\left(t_{2}-t_{1}\right)} z_{1}, w_{2}\right)$. Si definimos el control $v(\cdot)$ por la expresión $v(s):=v_{1}\left(s-t_{1}\right)$ para $s \in\left[t_{1}, t_{2}\right]$, es fácil verificar que la trayectoria $y(\cdot)$ correspondiente a $v(\cdot)$ y con condición inicial $y\left(t_{1}\right)=z$ satisface las condiciones afirmadas.

Definición 6. Diremos que el sistema (1) es R-auto-accesible si todo vector $w \in R$ es auto-accesible.

El siguiente resultado es consecuencia inmediata del teorema anterior

Corolario 3. Considere las siguientes condiciones :

(a) El sistema (1) es R-auto-accesible.

(b) El sistema lento es auto-accesible.

(c) $\operatorname{lm}\left(A_{1}\right) \subseteq<A_{1}, B_{1}>$.

(d) $\operatorname{rang}[\lambda E-A, B]=n$, para todo $\lambda \in \mathrm{C}, \lambda \neq 0$.

Entonces $(a) \leftrightarrow(b) \leftrightarrow(c) \rightarrow(d)$.

Un problema fundamental en teoría de control es el relativo a la estabilizabilidad del sistema. A continuación estudiaremos algunas relaciones entre autoaccesibilidad y estabilidad. Si denotamos por $\sigma(E, A):=\{\lambda \in \mathrm{C}: \operatorname{det}(\lambda E-A)=$ $0\}$ el espectro del par $(A, E)$ entonces [3] el sistema (1) es estable si, y solamente si, $\sigma(E, A) \subseteq \mathbf{C}^{-}$, donde $\mathbf{C}^{-}:=\{\lambda \in \mathbf{C}: \operatorname{Re}(\lambda)<0\}$. Por otra parte, el sistema (1) se llama estabilizable si existe una realimentación del tipo $u=F x+v$ tal que 
el sistema

$$
E \dot{x}(l)=(A+B F) x(t)+B v(\ell)
$$

es estable (esto incluye la condición de regularidad).

Del teorema 3.1-2 en [3] se obtiene la siguiente propiedad.

Proposicion 1. Si el sistema (1) es auto-accesible entonces el sistema (3)-(5) es estabilizable.

Demostración. Es suficiente observar que el sistema formado por las ecuaciones (3) y (5) es controlable

La auto-accesibilidad junto a la estabilidad nos ofrecen también un criterio de controlabilidad.

Proposicion 2. Si el sistema (I) es auto-accesible (respectivannente, R-autoaccesible) y estabilizable entonces (1) es controlable (resp., R-controlable).

Demostración. Existe una matriz $\mathrm{F}$ tal que $\sigma(E, A+B F) \subseteq \mathrm{C}^{-}$. Además, como

$$
\operatorname{rang}[\lambda E-A-B F, B]=\operatorname{rang}[\lambda E-A, B], \forall \lambda \in \mathrm{C},
$$

entonces $\operatorname{rang}[\lambda E-A, B]=n$, para todo $\lambda \in \mathbf{C}$. La afirmación es ahora consecuencia del teorema 1-(iii) y del Corolario 3 .

Por otra parte, es claro de la representación estandard del sistema (1) que $\operatorname{rang}(E)=n_{1}+\operatorname{rang}(N)$. A continuación siempre supondremos que $n_{11} \geq 1$.

Teorema 4. Si el sistema (1) es auto-accesible, entonces para cualquier conjunto simétrico $\Lambda \subseteq \mathrm{C}$ con $\operatorname{rang}(E)-n_{11}$ elementos, existe una matriz $\mathrm{F}$ tal que el sistema (6) tiene espectro $\sigma(E, A+B F)=\Lambda \cup\{0\}$.

Demostración. Procediendo como en la demostración del Corolario 1, el sistema (1) se puede descomponer en tres subsistemas con ecuaciones (3), (4), (5). El sub-sistema descrito por (5)-(3) es un sistema singular en el espacio de estado $X=Y_{2} \oplus \mathbb{R}^{n_{2}}$, determinado por las matrices

$$
E^{\prime}=\left[\begin{array}{cc}
I_{n_{12}} & 0 \\
0 & N
\end{array}\right] \text { y } A^{\prime}=\left[\begin{array}{cc}
A_{12} & 0 \\
0 & I
\end{array}\right]
$$

Por nuestros resultados previos este sistema es controlable y por el teorema 3-3.2 en [3], existe una aplicación lineal $K: X \rightarrow \mathbb{R}^{m}$ tal que $\sigma\left(E^{\prime}, A^{\prime}+B K\right)=\Lambda$. 
Si defininos $F: \mathbb{R}^{n} \rightarrow \mathbb{R}^{m 2}$ por $F\left(y_{1}+y_{2}+x_{2}\right)=h^{\prime}\left(y_{2}+x_{2}\right)$ entonem $\sigma(E) .1+$ $F F\}=\Lambda \cup\{0\}$

De manera similar, utilizando el teorema 3.3 .4 de [3] en lugar de] teorema 3-3.2, obtenetnos el sigurente resultado

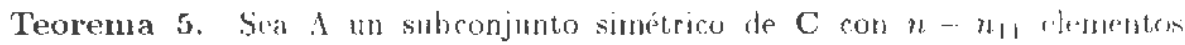
Si el sistema (1) es auto-accesible, entonces existen matrices $F_{1}$ y $F_{2}$ that qun. $\sigma\left(E+B F_{2}, A+B F_{1}\right)=\Lambda \cup\{0\}$

\section{Una propiedad geométrica de las trayectorias de auto-accesibilidard}

Sea $t_{1}>0$ y $u(\cdot)$ un control astrnisible definido en el intervalo $\left[0, t_{1}\right]$. Diremo que la trayectoria $x(\cdot)$ correspondiente al control $u(\cdot)$ es una trayectoria de aut.raccesibilidad si $x(0)=x\left(t_{1}\right)$. En la serción anterior hemos establecirlo cur uns sisterna singular puede retornar a sus estados auto-accesibles. Fin ista sorrinin mostraremus que las t rayertorias de anto-accesibilidad de un estado aulo-arresiblt no pueden escogerse atrbitrarianente pequenas.

Definición 7. Direnos que un vectur $\left(x_{1}, x_{2}\right)$ os un estado de equilitrio de:!

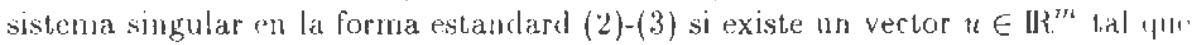
$A_{1} x_{1}+B_{1} u=0$ y $x_{2}+B_{2} u=0$.

El conjunto st formado por los estados de equilibro es un sulsespactice vectorial de $\mathbb{R}^{n_{1}}+\mathbb{R}^{n_{2}}$. si $s_{2}=\left(s_{1}, x_{2}\right)$ es un estado de equilibrio, entoncres escugiendo el control constante $u(t)=u$, la trayectoria correspondiente per rnantur. istariontiria en 2 .

A continuacion mostraremos que la integral de una trayectoria de intoaccesibilidad es un estado de equilibrio.

Proposición 3. Si $\mathrm{s}(\cdot)$ es ma trayectoria de auto- accesibilidad del sistema singular (2)-(3) en el intervalo $\left[0, t_{1}\right]$, entonces el estado

$$
y=\int_{0}^{t_{1}} x(s) d s
$$

pertenece a $S$.

Deimostración. . Como $x(t)=\left(x_{1}(t), x_{2}(t)\right)$ es una trayectoria de auto-accesibilidad del sistema $(2)-(3)$, entonces existe un control $u(\cdot) \in C_{p}^{h-1}$ definido en $\left[0, t_{1}\right]$ ta] que

$$
x_{1}(t)=e^{A_{1} t} w_{1}+\int_{0}^{t} e^{A_{1}(t-s)} B_{1} u(s) d s
$$




$$
x_{2}(t)=-\sum_{i=0}^{h-1} N^{i} B_{2} u^{(i)}(t),
$$

para todo $0 \leq t \leq t_{1}$ y donde $x(0)=x\left(t_{1}\right)=w=\left(w_{1}, w_{2}\right)$. Considerando que $x_{1}(t)$ es la trayectoria de un sistema normal, por los resultados establecidos en [5], podemos afirmar que

$$
A_{1} \int_{0}^{t_{1}} x_{1}(t) d t=-B_{1} \int_{0}^{t_{1}} u(t) d t .
$$

Por otra parte. de la expresión para $x_{2}(t)$ y del hecho que $N^{h}=0$ deducimos

$$
\begin{aligned}
\int_{0}^{t_{1}} x_{2}(t) d t & =-\sum_{i=0}^{h-1} N^{i} B_{2} \int_{0}^{t_{1}} u^{(i)}(t) d t \\
& =-B_{2} \int_{0}^{t_{1}} u(t) d t-N\left[\sum_{i=1}^{h-1} N^{i-1} B_{2}\left(u^{(i-1)}\left(t_{1}\right)-u^{(i-1)}(0)\right)\right] \\
& =-B_{2} \int_{0}^{t_{1}} u(t) d t .
\end{aligned}
$$

lo cual completa la demostración.

Para establecer nuestro próximo resultado introduciremos previamente algunas notaciones. Hemos mencionado anteriormente que existen transformaciones de coordenadas $P$ y $Q$ que permiten descomponer el sistema (1) en los subsistemas (2) y (3). Supongamos que $P$ es la matriz que efectúa la transformación $x=P\left(x_{1}, x_{2}\right)$. Si $x \in \mathbb{R}^{n}$ denotaremos por $\rho(x)$ a la distancia de $\left(x_{1}, x_{2}\right)$ al subespacio $S$, definida por

$$
\rho(x):=d\left(\left(x_{1}, x_{2}\right), S^{\prime}\right):=\inf \left\{\left\|\left(x_{1}, x_{2}\right)-y\right\|: y \in S\right\}
$$

siendo $\|\cdot\|$ una norma en $\mathbb{R}^{n_{1}} \oplus \mathbb{R}^{n_{2}}$.

Téorema 6. Si $w$ es un estado auto-accesible en $\left[0, t_{1}\right]$ del sistema (1) y $x(\cdot)$ es una trayectoria admisible tal que $x(0)=x\left(t_{1}\right)=w$, entonces

$$
\max \left\{\|w-x(t)\|: 0 \leq t \leq t_{1}\right\} \geq \frac{1}{\left\|P^{-1}\right\|} \rho(w)
$$


Demostración. Definamos el vector $y=\frac{1}{t_{1}} \int_{0}^{t_{1}}\left(x_{1}(t), x_{2}(t)\right) d t$. Por la proposición anterior sabemos que $y \in S$ y como $y$ también pertenece a la adherencia de la envolvente convexa de la imagen de la función $\left(x_{1}(\cdot), x_{2}(\cdot)\right)$, entonces podenus establecer las siguientes desigualdades

$$
\begin{aligned}
d\left(\left(w_{1}, w_{2}\right), S\right) & \leq\left\|\left(w_{1}, w_{2}\right)-y\right\| \\
& \leq \sup \left\{\left\|\left(w_{1}, w_{2}\right)-z\right\|: z \in \overline{c\left(\operatorname{Im}\left(x_{1}(\cdot), x_{2}(\cdot)\right)\right)}\right\} \\
& =\max \left\{\left\|\left(w_{1}, w_{2}\right)-\left(x_{1}(t), x_{2}(t)\right)\right\|: 0 \leq t \leq t_{1}\right\}
\end{aligned}
$$

Considerando que

$$
\begin{aligned}
\|x(l)-w\| & =\left\|P\left[\left(x_{1}(l), x_{2}(l)\right)-\left(w_{1}, w_{2}\right)\right]\right\| \\
& \geq \frac{1}{\left\|P^{-1}\right\|}\left\|\left(x_{1}(t), x_{2}(t)\right)-\left(w_{1}, w_{2}\right)\right\|
\end{aligned}
$$

entonces

$$
\begin{aligned}
\max _{0 \leq t \leq t_{1}}\|x(t)-w\| & \geq \frac{1}{\left\|P^{-1}\right\|} d\left(\left(w_{1}, w_{2}\right), S\right) \\
& =\frac{1}{\left\|P^{-1}\right\|} \rho(w) .
\end{aligned}
$$

\section{REFERENCIAS}

[1] Baccioti, A.: Auto-acessibilité par familles symetriques de champs de vecteurs. Ricerche di Automatica, 7(1976), 189-197.

[2] Barbanti, L.: Linear Volterra-Stieltjes integral equations and control. Proc. Equadiff 82, Wurtzburg, 1982.

[3] Dai, L.: Singular control systems. Lectures Notes in Control and Information Sciences, 118, Springer-Verlag, Berlin, 1989.

[4] Henriquez, H.: Auto-acessibilidade de sistemas de controle lineares em espaços de Banach. Anais do $1^{\circ}$ Congreso Latino-Americano de Automática, Campina Grande, Brasil, Vol. 3(1984), 860-865.

[5] Henriquez, H.; Rodriguez, A.; Castillo, G.: A geometric property of control systems with state in a Banach space. Systems and Control Letters, 8(1987), 225-229.

[6] Kailath, T.: Linear Systems Prentice-Hall, Englewood Cliffs, N.J., 1980. 
Recibido: 20 Novimbre de 1992.

Hernản Henriquez $\mathrm{M}$.

Departamento de Matenáticas y Ciencias de la Computación

Universidad de Santiago de Chile

Casilla 5659, Correo 2. Santiago. CHite

Genaro Castillo $\mathrm{G}$.

Departamento de Matemática

U'niversidad de Talca

(ampus Lircay, Talca, Ghile 